\title{
Education Management Information System in the Schools/Colleges - A Case Study of Tamilnadu Division
}

\author{
S. Shereen Priscila ${ }^{1 *}$, N. Venkatesan ${ }^{2}$ \\ ${ }^{1} P G$ Scholar, Department of Computer Science and Engineering, Sri Vidya College Engineering and \\ Technology, Virudhunagar, India \\ ${ }^{2}$ Assistant Professor, Department of Computer Science and Engineering, Sri Vidya College Engineering and \\ Technology, Virudhunagar, India \\ *Corresponding author: shereensolomon96@gmail.com
}

\begin{abstract}
Generating a Report collected from a survey using Laravel provides targeted insights in to the Parents/Students perspectives and can be used to guide an organizational strategy that helps the Management to develop or maintain a competitive advantages. It's helps to analyze Parents/Students experiences optimisation is to help Management for maximizing their longterm profitability through reaping the "lifetime value" and also for academic growth. The admission count report has an authority to uploaded in the Management server of the particular Institutions on the same time Parents/Students didn't get aware of this. The Management refused to reveal the exact admission count. It will confuse them to choose the better Institution. Collecting a feedback details from the old students and their parents by conducting survey that are already done by developers. The Parents/Students feedback data has been retrieved from online survey under Data Collection. The survey details are downloaded under four important status. The reviews and ratings are done by using these statuses for every Institutions. By using a single website, you can easily find the status of an Institutions (School, Colleges, University, Medical Board, etc.). Maintaining a Dashboard is an essential part for Parents/Students to utilize the growth of an organization by feedback rating. It is maintained as weekly, monthly and yearly-wise manner.
\end{abstract}

Keywords: Data collection, Dashboard, Laravel, Report generation.

\section{Introduction}

An educational institution is a common environment where people gain an education, including preschools, childcare, primary-elementary schools, secondary-high schools, and universities. They provide a large variety of learning environments and learning space. In India, Tamil Nadu is one of the most literate states. The state's literacy rate is calculated as $80.33 \%$ in 2011 , which is above the national average. A survey conducted by the Industry body Assocham ranks Tamilnadu top among Indian states with about 100\% GER in primary and upper primary education. The structure of Education in the state is based on the national level pattern with 12 years of schooling which is $10+2+3$, which elaborated as eight years of elementary education, five years of primary and three years of middle school education for the particular age groups of 6-11 and 11-14 years, respectively, followed by secondary and higher Secondary education of two years each besides two years of pre-primary education. Pre-primary class age group is classified as 3 to 4 . The higher secondary school certificate for students to pursue their studies either in universities or in colleges for higher education in general academic courses and also in technical and professional courses.

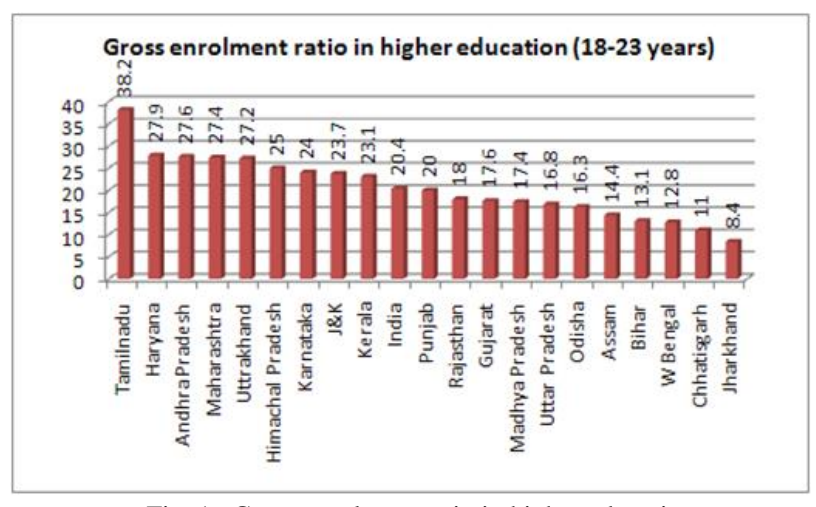

Fig. 1. Gross enrolment ratio in higher education

A college and universities are an Educational Institution or a constituent part of one. A college may be degree-awarding Tertiary Educational Institution, a part of federal university, an Institution offering vocational education, or a secondary school. Two types of universities in Tamilnadu are considered as Public University or Government University. It is run by State Governments of Union of India or Government of Union of India and Private University or Deemed University. The modern system of education was heavily influenced by the British starting in 1835.In India, the term "college" is commonly reserved for institutions that offer high school diplomas at year 12, and those that offer the bachelor's degree; 
some colleges, however, offer programmes up to $\mathrm{PhD}$ level. Generally, colleges are located in different parts of a state and all of them are affiliated to a regional university. The colleges offer programmes leading to degrees of that university. Colleges may be either Autonomous or non-autonomous. Autonomous Colleges are empowered to establish their own syllabus they conduct and assess their own examinations. In non-autonomous colleges, examinations are conducted by the particular universities at the same time for all colleges under its affiliation must obey them. There are several hundred universities and each university has affiliated colleges, often a large number.

The reason for writing a survey report is to analyse a research topic thoroughly, and to understand and evaluate the existing studies in an organized manner. In any research project it is an important step. After conducting a survey, we have to write the survey report. A survey report describes a survey, its results, and any patterns or trends found in the survey. Most survey reports follow a standard organization, broken up under certain headings. Each section has a specific purpose. Fill out each section correctly and proof read the paper to create a polished and professional report.

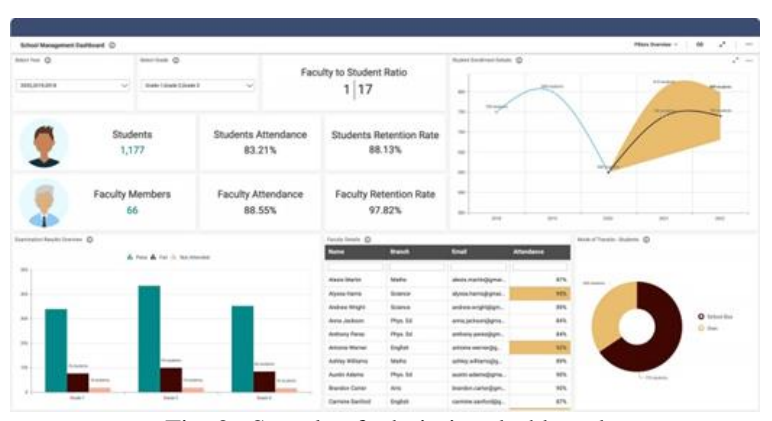

Fig. 2. Sample of admission dashboard

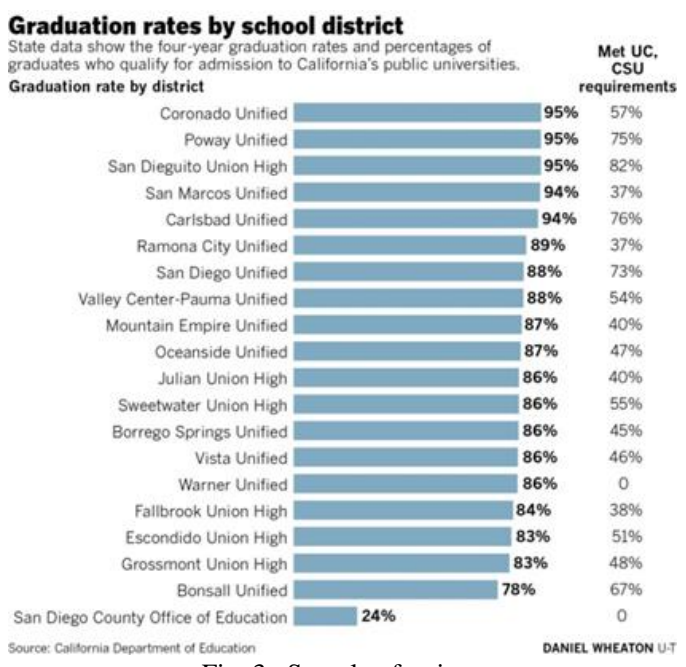

Fig. 3. Sample of ratings

\section{Literature Survey}

After conducting a survey, we have to generate a report for a survey. A survey report describes about survey, its results and any patterns found in the survey. Traditional written survey reports are a one-off snapshot of survey results. In that, we found the poor quality of survey reports published earlier. The two main primary problems are inefficient reporting of sufficiently rigorous survey research and poorly designed survey report affects the report quality.

The important reason for writing a survey report is to study a research topic completely, and to summarize the existing studies in an organized manner. It is an important step in any research project. Once you have finished conducting a survey, all that is left to do is write the survey report. A survey report describes a survey, its results, and any patterns or trends found in the survey. Most survey reports follow a standard organization, broken up under certain headings. Each section has a specific purpose. Fill out each section correctly and proofread the paper to create a polished and professional report.

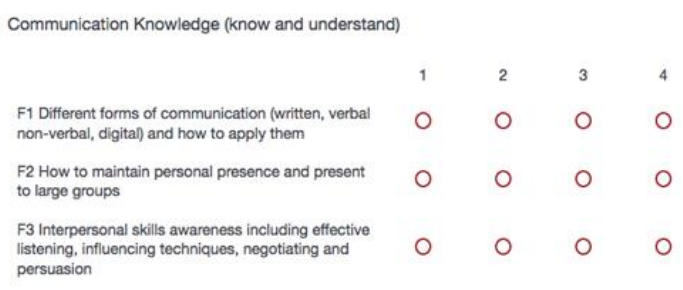

Fig. 4. Sample questionnaire

A report is written for a clear purpose and to a particular audience. Specific information and evidence are presented, analysed and applied to a particular problem or issue. The Information is presented in a clearly structured format making use of sections and headings so that the information is easy to locate and follow. For example, if you are asked to write a report you will usually be given a report brief which provides you with instructions and guidelines. The report brief may outline the purpose, audience and problem or issue that your report must address, together with any specific requirements for format or structure. This guide offers a general introduction to report writing, be sure also to take account of specific instructions provided by your department.

There will be confusion between parents and students to choose a better institution because of management's fake statement about admission count and rating. This will be done exactly by report generation. The main objective of this project is to design a framework for receiving an exact admission count and rating that are maintaining in a dashboard.

\section{Research Methodology}

In this paper, we discuss about the data retrieval from the online survey. After retrieving the data from online survey we have to generate the report for particular details. Once report has been generated, the survey report will be uploaded to the owner site. From this, owner receives the details about their product. At the same time, Partner also receiving the survey 
report. In this, they didn't get survey details just they receive the survey count to avoid forgery. Websites are built for human consumption not for machines. Getting that data from a website is vital to the success of your business. In fact, the internet is the largest source of business data on earth and it's growing by the minute. In this paper, we are collecting a data from same website. The project developer creates a survey for a customer or clients to know the feedback about the product. By using the same project name as a data we are going to collect the exact report of the survey status.

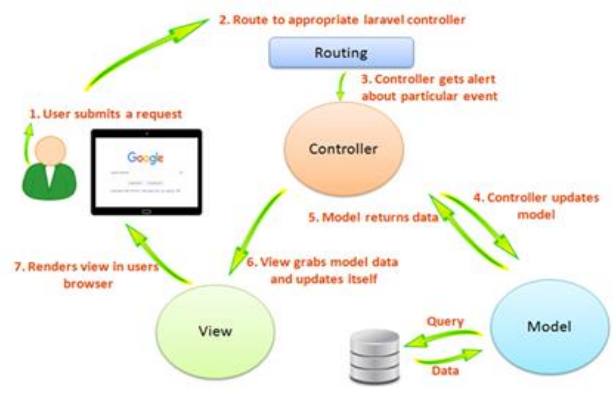

Fig. 5. MVC architecture for data collection

Representational State Transfer or RESTful web services are one way of providing interoperability between computer systems on the internet. This services allow requesting systems to access and manipulate textual representations of web resources using a uniform and predefined set of stateless operations. Also in computer programming, an application programming interface (API) is a set of subroutine definitions, protocols and tools for building applications. A good API makes it easier to develop a program by providing all the building blocks, which are then put together by the programmer. Therefore, a RESTful API is an application program interface that uses HTTP requests to GET, PUT, POST and DELETE data. For this project, I would be using the Laravel to develop the RESTful API project.

\section{Result and Experiment}

First, we are creating login page from a management website. We are having an authority to access this page from their site. By using an email id and password just login into our page.

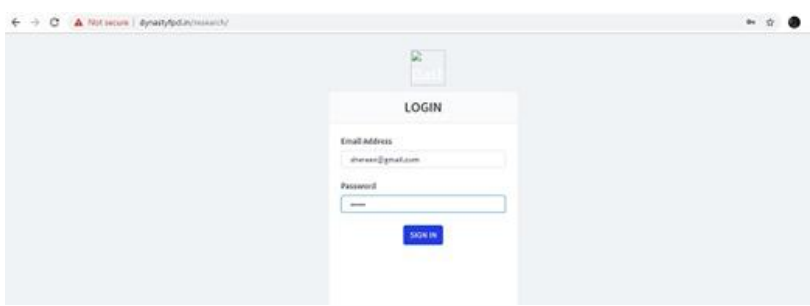

Fig. 6. Login page
This is our data collection page. Here we are extracting data from online survey that was already created by someone. It is the home page of our project.

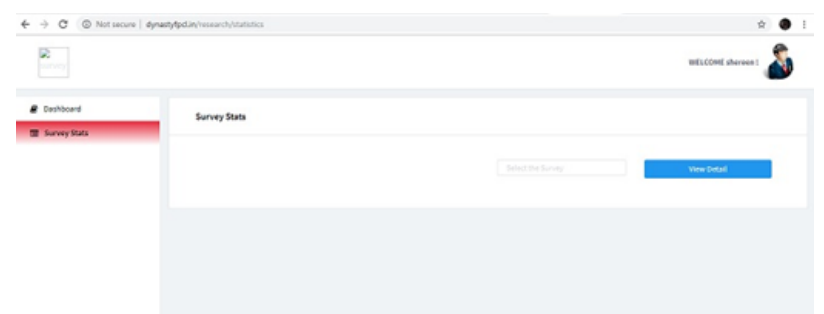

Fig. 7. Data collection page

In this case, suppose we entered a wrong data which is not created by the developer means it shows an error. It will not lead us to access the next page from a current page. We have to enter the correct survey name.

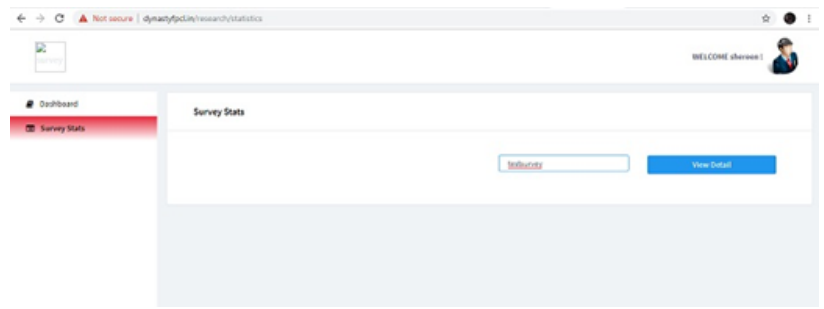

Fig. 8. Wrong data entry

If we enter an exact data then it will display the database order. From that we have to select our project to analyze the result for that particular survey.

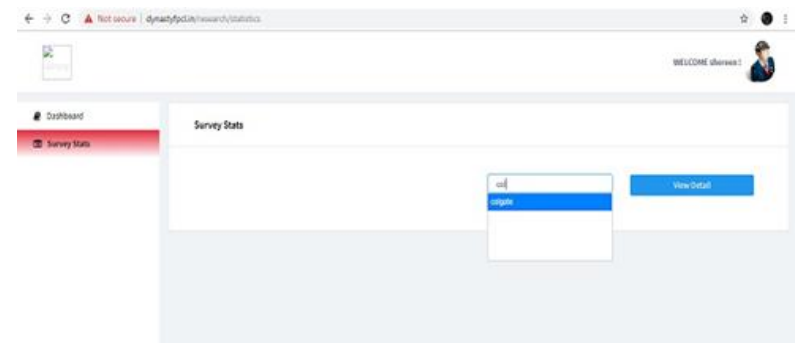

Fig. 9. Exact data entry

If we enter the exact data from the online database then the color will automatically change from blue to red which implies that we have an authority to open that survey.

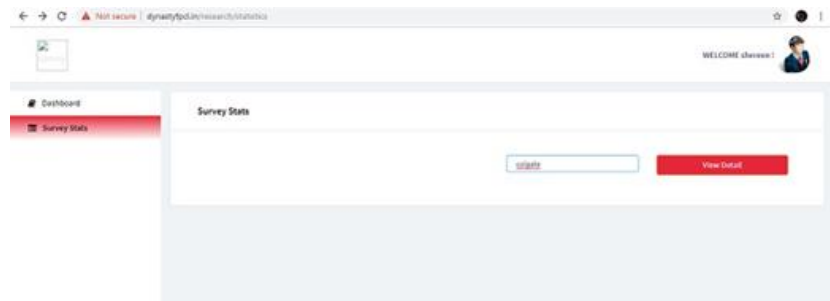

Fig. 10. Exact survey name 
This is an important page for our project. From this we can able to check the status for the particular survey. These are the four kind of status. The Incidence rate and Average Lack of Intent also calculated here.

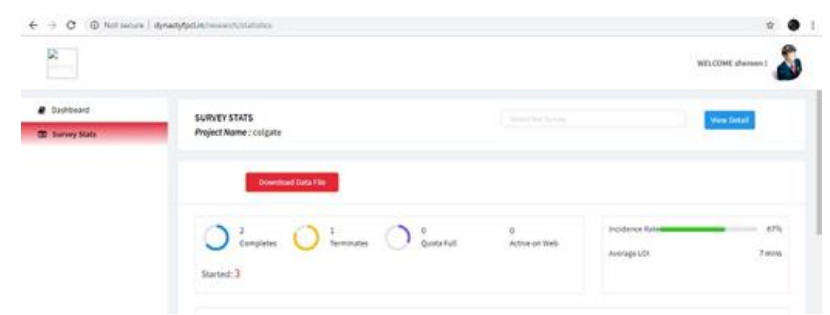

Fig. 11. Status of the survey

The next process is to download the particular data file for receive the result. These are done by the management.

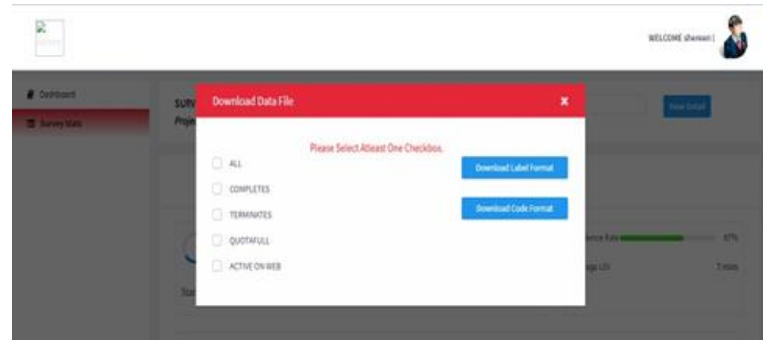

Fig. 12. Download data file

From this checklist we have to select anything that we want to know the details of that particular one. The four statuses are listed as a check box. If we select any one or all and then click the Download Label Format after that it started downloaded.

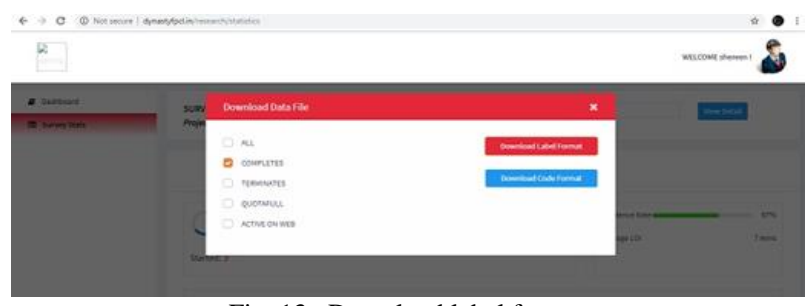

Fig. 13. Download label format

The Survey details about parents/students feedback have been uploaded to both the server. From this, She/he able to receive all the details related to that particular survey. It will be in csv file format.

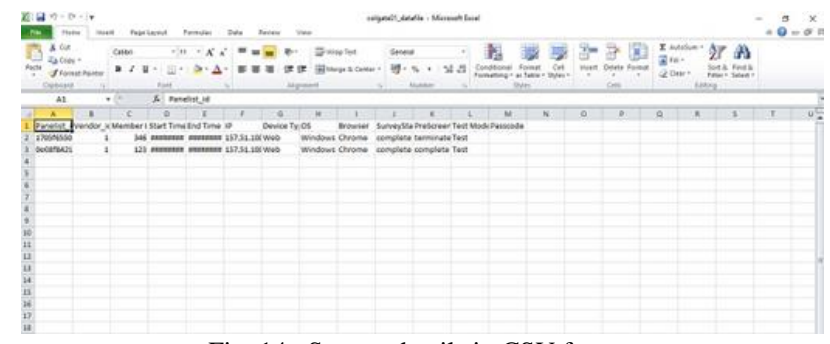

Fig. 14. Survey details in CSV format
For every institution, it's an important job to maintain their dashboard properly because it will lead a good representation of our effort. The graphical representation shows an exact result of our process.

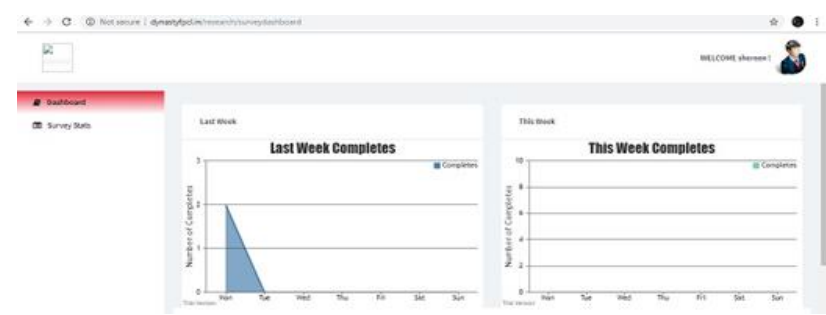

Fig. 15. Dashboard maintenance

There are different type of dashboard representation are available. We are using Line-Graph, Pie-Chart and Bar-chart. It will show a weekly, monthly and yearly-wise survey report.

A dashboard is a type of graphical user interface (GUI) which often provides view of key Performance Indicators (KPIs) relevant to a particular objective or business process. In other usage, "dashboard" is also known as "progress report" or "report". The "dashboard" is often displayed on a web page which is linked to a database that allows the report to be constantly updated.

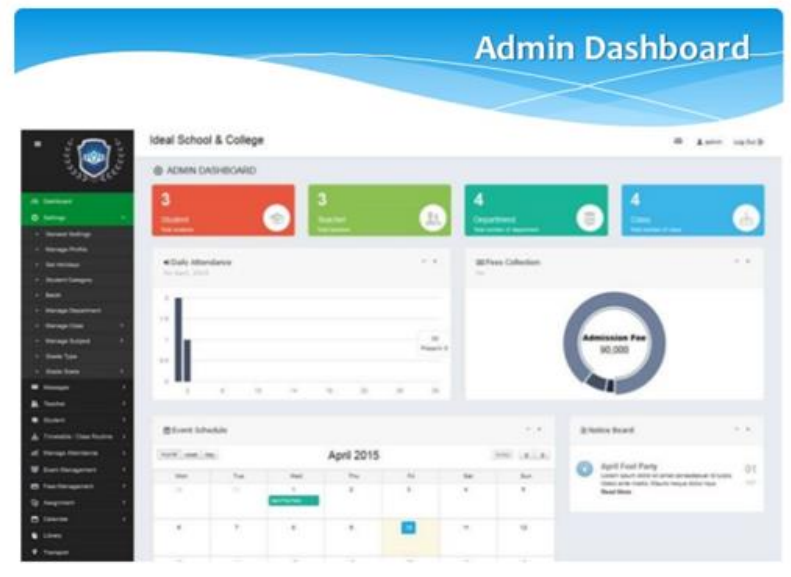

Fig. 16. Admin dashboard

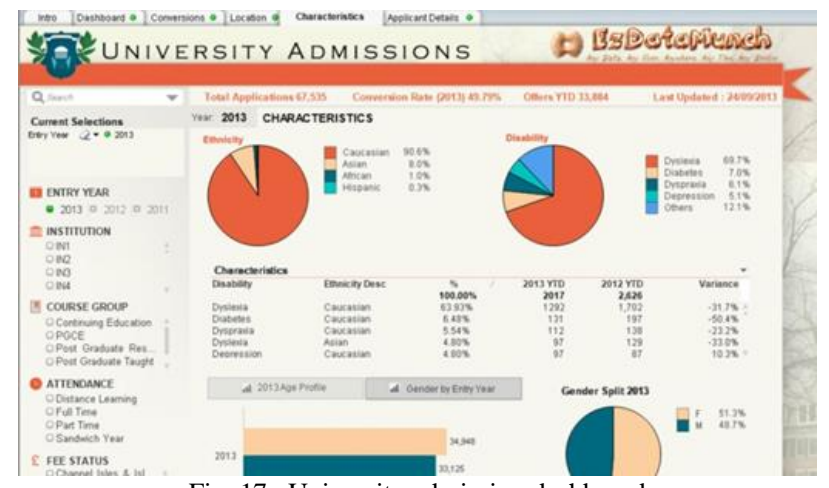

Fig. 17. University admission dashboard 
Volume-3, Issue-9, September-2020

\section{IJRESM journals.resaim.com/ijresm | ISSN (Online): 2581-5792 | RESAIM Publishing}

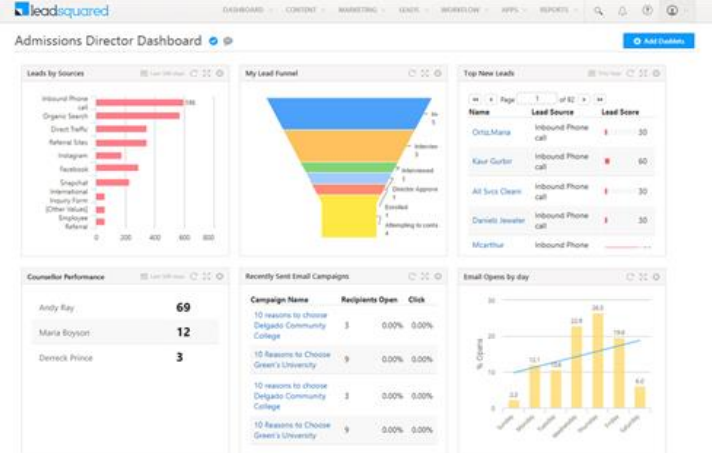

Fig. 18. Admission management dashboard

An educational institution appearance on a ranking list in the dashboard is entirely the result of what its own students/parents surveyed by our developers. Review reported about their campus experiences as well as how they rated various aspects of their college/school life. Our dashboard ratings are numerical scores on a scale of 60-99 that we give to all educational institution in the survey. We report those rating scores in eight categories, including Admissions Selectivity, Financial Aid, Fire Safety, and Green, which are based entirely on our student/parent surveys, our eight rating scores are based primarily on our surveys of administrators at the Institutions who annually provide us with institutional data we request about their Institutions. A few of our rating scores factor in some of our student survey data-e.g., our "Professors Accessible Rating" score is based on students' answers to our survey question that asks students to rate the accessibility of their professors on our five-point scale.

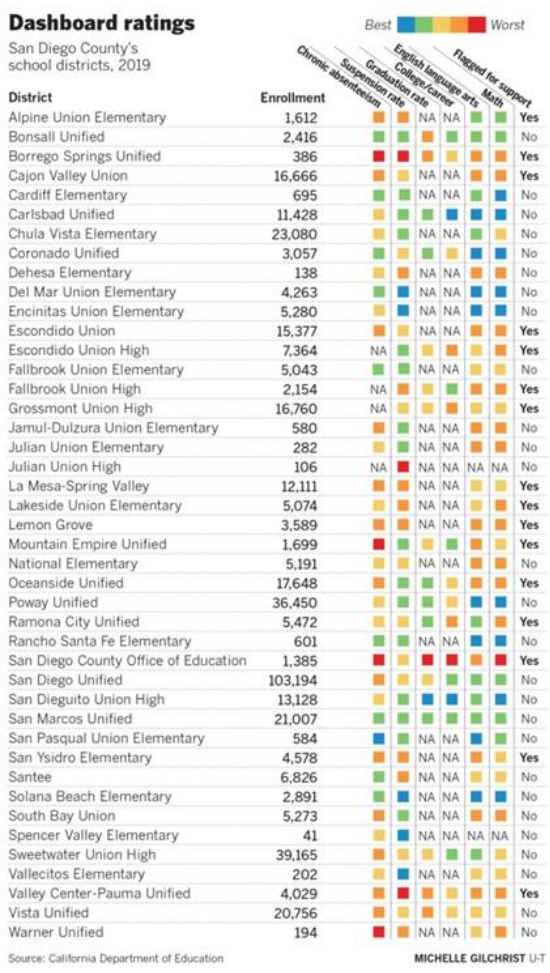

Fig. 19. Ratings

\section{Conclusion}

The result of the survey provides some of the experimental data about the perceptions of a sample of an Educational Institution Reports. The survey obtained information from the diverse group of Institutions. Based on the survey results and its analysis, depends on Parents/Students feedback about the Institutions conducted via survey with different question and rating session. By using their feedback and rating we are maintaining a dashboard which gives additional information to the society for choosing their best Institution. This will also useful to the Institution for receiving more number of admissions. Maintaining dashboard in the websites leads an efficient way to utilize the survey report details.

\section{References}

[1] Sagar SreeKumar Nair (2018), "A Survey report of the firefighters on fire hazards of PV fire", IEEE.

[2] Jingyu Zhang, Yefu Wu, Haojun Huang, Guolin Hou. (2018), “A New Human Eye Tracking Method Based on Tracking Module Feedback TLD Algorithm", IEEE.

[3] Kamaljit Kaur, Rajat Khurana (2018), "Deep Survey on Visual Object Tracking in Surveillance Environment “, IEEE

[4] Shantanu Dutta, Abdul Kaswar Tushar, Md Abdullah Al Mamun, Alvi Md. Ishmam, Sourav Kumar Mondal, Tarik Reza Toha, A.B.M. Alim Al Islam (2017), "Towards Perceiving and Resolving the Impediments to Reporting for the Developing Countries", IEEE

[5] Emitza Guzman, Mohamed Ibrahi, Martin Glinz. (2017), "Prioritizing User Feedback from Twitter: A Survey Report", IJLTEMAS.

[6] Suguru Ishizaki, Necia Werner, Stacie Rohrbach (2015), "Integrated system of learning resources for technical communication: A report on a student survey", IEEE.

[7] Isamu Kajitani, Takeshi Sakaguchi, Yoshio Matsumoto, Tamio Tanikawa , Testuo Kotoku (2015), "A Survey report on information costs in introducing technology to care services for older adults", IEEE.

[8] Lakshmi Dhandabani, Rajeev Sukumaran (2015)," Use of ICT in Engineering Education: A Survey Report", IEEE.

[9] Sun Jing-shui, Huang Qiu-hong (2013)," The Report of Questionnaire Survey on Chinese Resident's Income Gap Moderation", International Journal of Innovative Science and Research Technology.

[10] Khalid Masood, Jongyong Choi, Ricardo Gutierrez-Osuna (2012), "Consistency and Validity of Self-reporting Scores in Stress Measurement Surveys", International Journal of Innovative Research in Computer and Communication Engineering.

[11] Reiko Yamada (2012), "The Development of JCIRP: Self-reported Survey System to Assess Student Learning Outcomes", IEEE.

[12] Guangyu Zhu, Bala Venkatesh (2010), "Survey and Report on Feed-in Tariff in Canada", International Research Journal of Engineering and Technology (IRJET).

[13] T. A. Majid, M. N. A. Azman, S. A. S. Zakaria, S. S. Zaini, A. S. Yahya, M. S. S. Ahamad, M. H. Hanafi (2010), "The Industrialized Building System (IBS) Survey Report 2008- Educating the Malaysian Construction Industry", International Journal of Advanced Research in Electrical, Electronics and Instrumentation Engineering.

[14] Yin Ming, Wang Xuefeng, Sun Ming (2010), "On the web-based Tracking Survey of Educational Quality of Applied and Practical Majors", International Research Journal of Engineering and Technology (IRJET).

[15] Lin Liu, Tong Li, Fei Peng (2010), "Why Requirements Engineering Fails: A Survey Report from China", IEEE.

[16] Ed Bevers, Bob Saint (2006), "NRECA Reliability Reporting Survey and the New RUS Interruption Reporting Requirement", IEEE.

[17] V. J. Gosbell, S. Perera, R. Barr, A. Baitch (2004), "Primary and Secondary Indices for Power Quality (PQ) Survey Reporting", International Research Journal of Engineering and Technology (IRJET).

[18] K. Nishi, S. Shiraishi, M. Hamano, Y. Umeki, H. Kume (2004), "Survey Report of offshore wind conditions in prospects of offshore wind energy 
Volume-3, Issue-9, September-2020

journals.resaim.com/ijresm | ISSN (Online): 2581-5792 | RESAIM Publishing

conversion systems in Japan", International Journal of Advanced Research in Electrical, Electronics and Instrumentation Engineering.

[19] Michael F. Gard (2002), "A Status Report on Environmental Monitoring”, International Research Journal of Engineering and Technology (IRJET).

[20] V. J. Gosbell, Alex Baitch, Mathias H. J. Bollen (2010), "The Reporting of Distribution Power Quality Surveys", IEEE. 